\title{
SUBHARMONICITY, THE DISTANCE FUNCTION, AND $a$-ADMISSIBLE SETS
}

\author{
STEPHEN J. GARDINER
}

(Communicated by Clifford J. Earle, Jr.)

\begin{abstract}
Let $\Omega$ be an open subset of $\mathbb{R}^{n}$. The main result of this paper shows that a one-sided control on the curvature of $\partial \Omega$ is equivalent to subharmonicity of a function related to $\operatorname{dist}(X, \partial \Omega)$. An application is given.
\end{abstract}

\section{Results}

Throughout this paper $\Omega$ denotes a nonempty proper open subset of Euclidean space $\mathbb{R}^{n}(n \geq 2)$. Let $\bar{\Omega}$ and $\partial \Omega$ denote the closure and boundary of $\Omega$ respectively, and define

$$
u(X)= \begin{cases}\operatorname{dist}(X, \partial \Omega) & (X \in \Omega), \\ -\operatorname{dist}(X, \partial \Omega) & \left(X \in \mathbb{R}^{n} \backslash \Omega\right) .\end{cases}
$$

The following result is due to Armitage and Kuran [1]. (Only connected sets $\Omega$ were considered in [1], but the proof does not require connectedness.)

Theorem A. The set $\Omega$ is convex if and only if $u$ is superharmonic in $\mathbb{R}^{n}$.

In this paper we show that an analogous result holds when convexity is replaced by one-sided control on the curvature of $\partial \Omega$. Let $B(X, r)$ denote the open ball of center $X$ and radius $r>0$. An inner maximal ball (of radius $r$ ) is a ball $B(X, r) \subseteq \Omega$ such that $r=u(X)$. Such a ball touches $\partial \Omega$ in the sense that $\partial B(X, r) \cap \partial \Omega$ is nonempty. Let $a>0$. Following Kuran [2] we say that $\Omega$ is $a$-admissible if every inner maximal ball of radius $r<a$ is contained in an inner maximal ball of radius $a$. If $\Omega$ is $a$-admissible, then every point of $\partial \Omega$ is touched by an inner maximal ball of radius $a$, but not conversely (see $[2, \S 5])$.

Let $\phi_{n}:(0, \infty) \rightarrow \mathbb{R}$ be given by $\phi_{n}(t)=t^{2-n}$ if $n \geq 3$, and by $\phi_{2}(t)=$ $\log (1 / t)$. Our main result is stated below.

Theorem 1. The set $\Omega$ is a-admissible if and only if the function $X \mapsto$ $\phi_{n}(a-u(X))$ is subharmonic on the set $\{X: u(X)<a\}$.

Received by the editors December 29, 1989.

1980 Mathematics Subject Classification (1985 Revision). Primary 31B05; Secondary 26B25. 
As an application of the "only if" part of Theorem 1 we obtain a short proof of the following, which is the main result in [2].

Corollary. Let $\Omega$ be bounded and a-admissible, and let $v$ be a positive superharmonic function on $\Omega$. Then there is a positive constant $k$ such that $v \geq k u$ on $\Omega$.

To see this, we note that $v$ has a positive lower bound, $K$ say, on the compact set $\{X: u(X) \geq a / 2\}$. By the minimum principle we have

$$
v(X) \geq K \frac{\phi_{n}(a-u(X))-\phi_{n}(a)}{\phi_{n}(a / 2)-\phi_{n}(a)} \geq K \frac{\max \{1, n-2\} a^{1-n}}{\phi_{n}(a / 2)-\phi_{n}(a)} u(X)
$$

on the set $\{X: 0<u(X)<a / 2\}$. Since $u$ is bounded above, by $L$ say, on $\Omega$, we have $v \geq(K / L) u$ on $\{X: u(X) \geq a / 2\}$. Thus the corollary is proved.

It is shown in $[2, \S 5]$ that the corollary fails if the hypothesis of $a$-admissibility is weakened slightly to the condition that every $Z \in \partial \Omega$ is touched by an inner maximal ball of radius $a$.

\section{Proof of TheOREM 1}

2.1. We begin with the "only if" part of the theorem. For each $Y \in \mathbb{R}^{n}$ define $s_{Y}(X)=a-|X-Y|$. Let $\Omega$ be $a$-admissible and let $E=\{Y: B(Y, a) \subseteq \Omega\}$. It is clear that $u \geq \sup _{Y \in E} s_{Y}$ on $\mathbb{R}^{n}$. To see that equality holds on the set $\{X: u(X)<a\}$, we consider the following two cases.

(i) If $u(X) \leq 0$, there is a point $Z \in \partial \Omega$ such that $u(X)=-|X-Z|$. As observed in $\S 1$, there is a ball $B(P, a) \subseteq \Omega$ which touches $\partial \Omega$ at $Z$. Hence $u(X)=s_{P}(X)$.

(ii) If $0<u(X)<a$, the inner maximal ball $B(X, u(X))$ is contained in another inner maximal ball $B(P, a)$. Hence, again, $u(X)=s_{P}(X)$.

Since the function $X \mapsto \phi_{n}\left(a-s_{Y}(X)\right)$ is harmonic on $\mathbb{R}^{n} \backslash\{Y\}$, it follows that the continuous function $\phi_{n}(a-u(\cdot))=\sup _{Y \in E} \phi_{n}\left(a-s_{Y}(\cdot)\right)$ is subharmonic on $\{X: u(X)<a\}$.

2.2. The "if" part of the proof uses ideas from Armitage and Kuran [1] and Parker [3]. Let $\mathscr{M}(f ; X, r)$ denote the mean of a function $f$ over $\partial B(X, r)$. We begin by recording two lemmas.

Lemma A. Let $Y, Z_{1}, Z_{2}$ be distinct points in $\mathbb{R}^{n}$ such that $\left|Y-Z_{1}\right|=\mid Y-$ $Z_{2} \mid=d<a$, and let $v(X)=\min \left\{\left|X-Z_{1}\right|,\left|X-Z_{2}\right|\right\}$. Then there exists $r_{0} \in$ $(0, a-d)$ such that $\mathscr{M}\left(\phi_{n}(a-v(\cdot)) ; Y, r\right)<\phi_{n}(a-v(Y))$ for all $r \in\left(0, r_{0}\right)$.

Lemma B. Let $B(X, r) \subset \Omega$ be such that $\partial B(X, r) \cap \partial \Omega=\{Z\}$ and let $Q \in \overline{B(X, r)} \backslash\{Z\}$. Then there exists $\varepsilon_{0}>0$ such that $\overline{B(X+\varepsilon(Q-Z), r)} \subset \Omega$ for all $\varepsilon \in\left(0, \varepsilon_{0}\right)$.

Lemma $A$ is a modified version of [1, Lemma 2] and can be proved by similar reasoning. A sketch proof of Lemma B can be found in [3, p. 504].

Now let $\Omega$ be such that the function $\phi_{n}(a-u(\cdot))$ is subharmonic on the set $\{X: u(X)<a\}$. Let $0<r<a$, let $B\left(X_{0}, r\right)$ be an inner maximal ball, 
and choose $Z_{0} \in \partial B\left(X_{0}, r\right) \cap \partial \Omega$. We are going to show that there is an inner maximal ball $B\left(Y_{0}, a\right)$ containing $B\left(X_{0}, r\right)$.

Let $Y \in B\left(Z_{0}, a\right) \cap \Omega$ and suppose that there are distinct points $Z_{1}, Z_{2} \in \partial \Omega$ such that $\left|Y-Z_{1}\right|=\left|Y-Z_{2}\right|=u(Y)$. Let $v$ be as in Lemma A. Since $u(Y) \leq\left|Y-Z_{0}\right|<a$, we can choose $r_{0}$ as in Lemma A. For $0<\rho<r_{0}$ we have

$\mathscr{M}\left(\phi_{n}(a-u(\cdot)) ; Y, \rho\right) \leq \mathscr{M}\left(\phi_{n}(a-v(\cdot)) ; Y, \rho\right)<\phi_{n}(a-v(Y))=\phi_{n}(a-u(Y))$, contradicting the fact that $\phi_{n}(a-u(\cdot))$ is subharmonic on a neighborhood of $Y$. Hence every point in $B\left(Z_{0}, a\right) \cap \Omega$ has a unique closest point in $\partial \Omega$.

Let $X_{\varepsilon}=X_{0}+\varepsilon\left(X_{0}-Z_{0}\right)$ and $B_{\varepsilon}=B\left(X_{\varepsilon}, r\right)$ for $\varepsilon>0$. By Lemma B there is an $\varepsilon_{0}>0$ such that $\bar{B}_{\varepsilon} \subseteq \Omega$ for all $\varepsilon \in\left(0, \varepsilon_{0}\right)$. Now fix $\varepsilon \in\left(0, \varepsilon_{0}\right)$ and let $r_{\varepsilon}=\sup \left\{\rho \in[r, a]:\right.$ there exists $Y$ for which $\left.B_{\varepsilon} \subseteq B(Y, \rho) \subseteq \Omega\right\}$.

Clearly $r<r_{\varepsilon} \leq a$. There is a sequence of balls $B\left(P_{m}, \rho_{m}\right)$ such that $\rho_{m} \rightarrow r_{\varepsilon}$ and $B_{\varepsilon} \subseteq B\left(P_{m}, \rho_{m}\right) \subseteq \Omega$ for each $m$. By choosing a convergent subsequence of $\left(P_{m}\right)$, with limit $P_{0}$ say, it follows that $B_{\varepsilon} \subseteq B\left(P_{0}, r_{\varepsilon}\right) \subseteq \Omega$.

Now suppose that $r_{\varepsilon}<a-\varepsilon r$. Since

$$
\left|P_{0}-Z_{0}\right| \leq\left|P_{0}-X_{\varepsilon}\right|+\left|X_{\varepsilon}-Z_{0}\right| \leq r_{\varepsilon}-r+(1+\varepsilon) r<a,
$$

there is a unique point $Q_{0}$ of $\partial \Omega$ that is closest to $P_{0}$. By the maximality of $r_{\varepsilon}$ we have $\left|P_{0}-Q_{0}\right|=r_{\varepsilon}$, and so $\partial B\left(P_{0}, r_{\varepsilon}\right) \cap \partial \Omega=\left\{Q_{0}\right\}$. Let $Q \in \partial B_{\varepsilon}$ be chosen so that $\left|Q-P_{0}\right|$ is maximized. Clearly $Q \neq Q_{0}$, since $\bar{B}_{\varepsilon} \subseteq \Omega$. By Lemma B there is a $\delta_{0}>0$ such that

$$
\overline{B\left(P_{0}+\delta\left(Q-Q_{0}\right), r_{\varepsilon}\right)} \subseteq \Omega, \quad\left(0<\delta<\delta_{0}\right) .
$$

For small $\delta>0$ we also have

$$
B_{\varepsilon} \subseteq B\left(P_{0}+\delta\left(Q-Q_{0}\right), r_{\varepsilon}\right) .
$$

But (1) and (2) together contradict the maximality of $r_{\varepsilon}$. Hence $a-\varepsilon r \leq r_{\varepsilon} \leq a$.

Thus, for each large $m \in \mathbb{N}$, there is a ball $B\left(Y_{m}, r_{m}\right)$ such that $a-r / m \leq$ $r_{m} \leq a$ and

$$
B_{1 / m} \subseteq B\left(Y_{m}, r_{m}\right) \subseteq \Omega .
$$

It follows that there exists a point $Y_{0}$ such that

$$
B\left(X_{0}, r\right) \subseteq B\left(Y_{0}, a\right) \subseteq \Omega,
$$

as required.

\section{REFERENCES}

1. D. H. Armitage and Ü. Kuran, The convexity of a domain and the superharmonicity of the signed distance function, Proc. Amer. Math. Soc. 93 (1985), 598-600.

2. Ü. Kuran, On positive superharmonic functions in a-admissible domains, J. London Math. Soc. (2) 29 (1984), 269-275.

3. M. J. Parker, Convex sets and subharmonicity of the distance function, Proc. Amer. Math. Soc. 103 (1988), 503-506. 